\title{
Aportaciones educativas de los Modelos informatizados de diagnóstico cognitivo en el algoritmo de la sustracción.
}

\author{
Ana Belén Sánchez García \\ asglusal es \\ Universidad de Salamanca
}

\section{Introducción}

Con el advenimiento del programa cognitivista, las teorías psicológicas se marcan como objetivo en el ámbito de los errores aritméticos, la descripción de los procesos que generan tales conductas erróneas. El propósito del diagnóstico, no va ser solamente el rendimiento o conducta final del alumno en una tarea determinada; sino la evaluación paso a paso del proceso que genera tal actuación. Esta nueva forma de abordar el diagnóstico es denominado por Ohlsson. S.; Langley, P. (1988, pp. 42), "Cognitive diagnosis". Para estos autores, el problema que conlleva este tipo de valoración, es un problema de investigación que radica en cómo evaluar el conocimiento o falta del mismo en los sujetos.

Inicialmente, vino auspiciado por las aportaciones de la Teoría de la Gestalt y de autores de tradición Piagetiana. Sin embargo, es en 1972, cuando Newell y Simon, diseñan un método que pretendía la construcción de un modelo que proporcionara información sobre los procesos de resolución de problemas, cuando realmente se configura como un campo de estudio aplicado a procesos matemáticos. Posteriormente, se vio reforzado por las aportaciones que, desde la inteligencia artificial, influyeron en la informatización del diagnóstico de los errores en la aritmética con desarrollos; cuyo objetivo principal era la detección y diagnóstico de los "bugs", o errores sistemáticos que surgen en el aprendizaje de procesos matemáticos básicos.

Por tanto, son las investigaciones de Newell y Simon, y las aportaciones de la inteligencia artificial que construye sistemas inteligentes, las que conforman incipientemente este nuevo diagnóstico de procesos matemáticos erróneos.

La plataforma de trabajo que utilizan estas investigaciones es el contexto o espacio del problema, que resulta esencial para la diagnósis; pues es el marco en el cuál efectúa su labor. Según (Ohlsson. S. \& Langley, P., 1988), el espacio del problema consiste en todas las situaciones, que pueden derivarse del estado inicial por las aplicaciones sucesivas de operadores, hasta llegar a lo que ellos denominan “criterio de la terminación” (Ohlsson. S. \& Langley, P., 1988, pp. 43).

Así, para realizar un diagnóstico cognitivo aplicado al algoritmo de la resta, hay que describir los procesos y subprocesos mentales que un sujeto muestra a través del espacio del problema algorítmico .Aunque, podemos pensar que, este análisis corresponde más al campo de la Psicología, sus resultados son de gran interés para el contexto educativo; pues nos permiten actuar sobre los procesos que abocan al sujeto a cometer el error.

En definitiva, consiste en definir un espacio del problema e investigar el camino de su solución paso a paso. Hemos de señalar que, para el contexto educativo, supone un trabajo añadido a la labor docente que resulta difícil de llevar a cabo de manera eficiente. Por tanto, y siguiendo a Olhson y Langley (1988:45), el uso del potencial del diagnóstico cognitivo, es impráctico a menos que pueda informatizarse. Así, ha de ser complementado para que sea eficaz con las aportaciones de las 
Tecnologías de la Información.

\section{Tratamientos informatizados: énfasis en el conocimiento procesal}

De la fusión entre los postulados teóricos de la ciencia cognitiva y la tecnología de la información, han nacido distintos modelos de indiscutible aplicación al diagnóstico, y tratamiento de las dificultades en el área de las matemáticas.

Posiblemente una de las mejores aportaciones, en este sentido, sea el desarrollo de simuladores que representan los procesos de aprendizaje que ha de llevar a cabo un estudiante con el fin de asegurar la consecución de un contenido determinado. Son por tanto, de gran ayuda para los docentes y para los propios estudiantes, en la tarea común de aprendizaje.

Antes de detenernos específicamente en los modelos de tratamiento diagnóstico de los errores en la sustracción, vamos a realizar una breve puntualización sobre los sistemas tutores inteligentes. ITSs (Intelligent Tutoring Systems), son sistemas informatizados de aprendizaje, relacionados con determinados dominios de conocimiento, y pueden ser definidos como el proceso de recoger y mantener la información con respecto al estado de conocimiento que presenta un aprendiz en relación a un tema o contenido concreto. Por lo que se refiere a la estructura de los ITS, básicamente existe un consenso en que su arquitectura estaría compuesta por tres componentes principales (Barr \& Feigenbaum, 1982; Bonnet, 1985, citados por Brusilovskiy, 1994): módulo de conocimiento experto, módulo del modelo estudiante y módulo tutor.

La representación del conocimiento es el problema más importante en el diseño del modelado del aprendiz en estos sistemas. La mayor parte de ellos, reproducen los diferentes tipos de competencias intelectuales como modelos de conocimiento declarativo o procesal.

Los modelos de conocimiento declarativo, muestran el conocimiento a través de un conjunto de hechos y reglas, que están organizados para que el sistema interactuando con el alumno, pueda razonar con ellos; mientras el conjunto de conocimiento es reconocido o aprendido por el usuario. Normalmente, la estructura de modelos de aprendizaje declarativos y la información compilada en los mismos están relacionadas con los objetivos de aprendizaje establecidos en los currícula normativos de la enseñanza obligatoria. Si para los modelos de conocimiento procesal se utilizan redes procesales, para la construcción de estos modelos se utilizan a menudo las redes semánticas.

El primero de los muchos que se han construido, fue SCHOLAR, cuyo sistema de representación está fundamentado en una red semántica. Esta red está compuesta por una serie de nodos que representan conceptos como ciudades de la geografía del sur de América. El sistema fue el primer ITS documentado en comunicación científica por Carbonell en 1970.Igualmente, podemos hacer referencia al sistema GRUNDY, que estudia y clasifica los diferentes tipos de personalidades en función de la autodescripción personal del propio usuario. Otro sistemas interesantes, incardinados dentro de los modelos de conocimiento procesal, basados en redes bayesianas y aplicados a la resolución de problemas en Física son, el sistema POLA de (Conatti y Vanlehn, 1996) o el sistema OLAE de (Martin,. y VanLehn, 1993).

Estos últimos modelos procesales, tratarían de representar el conocimiento a través de una red de procesos y subprocesos, en base a un conjunto de reglas o acciones relacionados con ellos. El conocimiento en el que inciden de manera directa, es el conocimiento que versa sobre los procesos que dirigen y secuencian la realización de determinadas tareas. El objetivo más inmediato de los mismos es lograr un nivel de descripción, que permita asociar la actuación del aprendiz directamente con los componentes individuales de la red procesal. Autores como (Dillenbourg y Self, 1992), afirman que, el elemento que conforma y representa a este modelo, es fundamentalmente que sus componentes no son independientes; sino que están relacionados los unos con los otros. Este arquetipo, es el que se ha encargado más eficientemente de los errores en tareas matemáticas; ya que tiene capacidades de diagnóstico muy importantes como describiremos en el apartado siguiente. Un 
sistema pionero basado en este modelo es BUGGY, diseñado por Brown y Burton, se configura alrededor de la red procesal del algoritmo de la resta.

\subsection{Los sistemas inteligentes y los errores que cometen los niños en la sustracción.}

Durante las últimas décadas, numerosos trabajos han ahondado sobre la naturaleza del error. La investigación realizada por Brown y Burton (1978) con el sistema informatizado "Buggy", constituye un referente esencial en la literatura científica sobre la cuestión. El sistema, era básicamente un simulador de aprendizaje que intentaba identificar y tratar los distintos bugs o errores producidos en el algoritmo de la resta. Los errores, se basaban en un modelo mental avalado por una teoría psicológica.

A partir de este primer estudio, comienzan numerosas investigaciones que tratan de abordar los errores en distintas habilidades cognitivas. Se realiza una amplia investigación sobre los tipos de errores, y se categorizan en catálogos o librerías que se incorporan a los programas informatizados, como reglas de producción específicas del sistema, que dirigen las interacciones entre el estudiante y el tutor.

El trabajo de VanLehn, extiende esta concepción de Buggy, y analiza cómo se generan estos errores (VanLehn, 1990). Para este autor, los bugs son el resultado infructuoso de intentar extender las reglas existentes, y aplicarlas a nuevas situaciones y esta acción conformaría lo que el denomina "reparaciones". Las reparaciones pueden programarse y pueden predecirse por la teoría del callejón sin salida que es aplicada para pronosticar los bugs de los estudiantes en la solución de restas multicolumna. Con el objetivo de establecer una librería de bugs, el autor, diseñó un sistema informatizado denominado SIERRA.

Otros autores importantes en cuanto al diseño de teorías psicopedagógicas, relacionadas con la adquisición del error algorítmico, que utilizan sistemas informatizados son Young y O’Shea (1981), con el sistema PS, (Sistema de Producción de Errores en la Sustracción), Ohlson y Langley con su sistema DPF (Diagnostic Path Finder, de 1988), y más recientemente, Langley, Cumings and Shapiro que han presentado recientemente, la arquitectura cognitiva jerarquizada denominada ICARUS, que comparte algunos rasgos centrales con sus predecesores e intenta diagnosticar los diferentes tipos de errores en la sustracción dentro de un espacio cognitivo simulado en el sistema informático.

\subsection{Modelos de conocimiento procesal que diagnostican y trabajan sobre los errores en la sustracción.}

VanLehn, K., Ohlsson, S., \& Nason, R. (1994), realizaron una interesante revisión en relación a las características más importantes de los simuladores utilizados en el ámbito educativo. En esta comunicación, sintetizaron algunas cuestiones importantes relacionadas con la repercusión a nivel instructivo de los simuladores. Los autores, hacen hincapié, en que estos simuladores permiten a los docentes ver cómo sus acciones educativas afectan al conocimiento de los estudiantes. Por otra parte, los estudiantes pueden aprender en colaboración con un estudiante simulado; porque el estudiante simulado puede representar el rol de un estudiante experto, y por último los docentes pueden probar sus programas en los estudiantes simulados.

De lo anteriormente expresado, podemos concluir que la tecnología relacionada con la simulación por ordenador de procesos de aprendizaje, ha revertido de manera positiva en la ciencia de la educación, pues podemos utilizar esta tecnología en cada una de las fases del acto educativo (programación, enseñanza, evaluación, y obtener buenos resultados (VanLehn, K., Ohlsson, S., \& Nason, R. 1994).

Durante estas últimas décadas, la existencia de investigaciones relacionadas con el aprendizaje humano y los procesos de adquisición del conocimiento, han influido de manera indiscutible en la utilización de nuevas metodologías, basadas en tecnologías de la información y la comunicación aplicadas a la enseñanza,(VanLehn, 1983a,). De estas investigación surgen teorías generales de adquisición del conocimiento, (Anderson, 1983, Holand, Holyoak, Nisbett y Thagard, 1986, Newell, 
1990; Ohlsson, 1993, VanLehn 1983b), citados por VanLehn, K., Ohlsson, S., \& Nason, R. (1994), que inciden en el uso de metodologías variadas dentro del aula.

Del nacimiento de teorías que avalan la construcción de sistemas informatizados que simulen a los estudiantes cuando aprenden, surgen simuladores aplicados al aprendizaje y diagnóstico de los errores en la aritmética (Ohlsson and Rees 1991, VanLehn 1990, Young y O’Shea 1981, Ohlsson, S., \& Langley P. (1988), etc. A continuación, describiremos resumidamente los modelos más importantes en el ámbito de la sustracción.

\section{Buggy, Debuggy, Idebuggy-.}

Con el propósito de responder al origen de los errores cometidos por los estudiantes en el aprendizaje del algoritmo de la sustracción, Brown y Burton en 1978 diseñaron el modelo " $B U G G Y$ ". Su objetivo prioritario, básicamente giraba en torno, a la construcción de un modelo diagnóstico para los errores que cometen los estudiantes durante el aprendizaje del algoritmo de la resta. "Buggy", pretendía ayudar a los docentes a comprender el proceso de aprendizaje de los errores para poder diagnosticarlos de manera eficiente. Se simulaba un problema cuya solución estaba presidida por un error, y se demandaba al profesor que resolviera el problema usando estos procedimientos incorrectos. Con dicha metodología, pretendía que los profesores se acercaran a los procesos internos subyacentes al error, que les permitiera un diagnóstico rápido, cuya finalidad última, fuera el conocimiento más exhaustivo de los procesos internos inherentes al mismo.

Después de Buggy, Brown y Vanlehn desarrollan en 1981, DEBUGGY, versión on line, e IDEBUGGY en 1982, versión interactiva. Estos diseños posteriores están basados en Buggy, siendo Idebuggy un sistema que agrega la capacidad de generar dinámicamente problemas para ayudar al diagnosticador a identificar errores.

En conclusión, siguiendo a Brown y Burton (1978), Buggy asume que, a menudo algunos comportamientos de los alumnos que parecen ser aleatorios tienen una explicación subyacente simple, inteligente y completa. Por tanto, un diagnóstico apropiado, puede dirigirse a remediar las debilidades específicas que presenta el alumno en tal habilidad. La importancia de admitir simplemente que pueden existir errores subyacentes que no aparecen de forma evidente, es interesante para la acción pedagógica. Sin la apreciación de este hecho, un maestro puede ver el error en un problema particular como un descuido, o fracaso total del algoritmo, e inmediatamente en algunos casos, experimentar una disminución considerable de las expectativas con respecto a la capacidad del estudiante.

\section{Modelo informatizado - "SIERRA”-.}

Debido a las limitaciones del sistema "Buggy", que no podía generar errores ("bugs") automáticamente, y el avance teórico que supuso la Teoría de la Reparación de Brown y VanLehn. Los autores, propusieron el modelo Sierra (VanLehn, 1987), que es el resultado de aplicar la formulación teórica de la teoría de la Reparación implementada como sistema informatizado. La teoría asume la explicación de los pasos implícitos en el aprendizaje de habilidades procedimentales, e intenta describir por qué, los niños cometen errores durante este aprendizaje. Es, por tanto, una interpretación sintáctica del proceso que fue implementada en forma de desarrollo informatizado con el programa Sierra. Para su diseño, los autores utilizaron los datos obtenidos a través del estudio que llevaron a cabo con niños que fueron testados en problemas aritméticos (Brown \& VanLehn, 1980).

El sistema Sierra es una simulación al igual que Buggy, que se usó como prueba diagnóstica y permitió observar los pasos que lleva a cabo el aprendiz en la adquisición de errores o bugs aritméticos. Simula paso a paso, procesos de aprendizaje de habilidades aritméticas, a través de ejemplos resueltos con el fin de modelar el proceso de aprendizaje aritmético. (VanLehn, 1983b,1987).

Los componentes del modelo consisten en un lenguaje interno de programación, basado en un tipo de 
razonamiento condicional, y la ejecución de los pasos a través del espacio del problema con el fin de obtener la solución.

Por tanto, los autores para diseñar el programa Sierra, utilizaron un lenguaje de representación que expresara el conocimiento sobre los procedimientos. El argumento se basaba en la idea que asume que con este lenguaje sintáctico, se podrían representar en el sistema cada uno de los pasos de adquisición del error, y de las posibles reparaciones que los niños acometían. Lo que a su vez, les permitía clarificar cuál es el proceso, y en qué punto se adquiere el bug o error durante el aprendizaje. Paralelamente a la presentación del procedimiento correcto de la resta, el sistema detallaba el proceso erróneo, que se fundamentaba en los cambios que se producían en el procedimiento correcto y las acciones llevadas a cabo desde la interface o externamente al sistema.

Esta acción, informaba sobre qué tipo de interpretación de la habilidad estaba ejecutando el sujeto, y qué clase de lenguaje de representación usaba en ese momento, lo que a nivel pedagógico era especialmente relevante.

\section{Sistema informatizado - "SOAR"}

Siguiendo a Walter Fritz (1997), podemos decir que la arquitectura informatizada Soar fue creada por John Laird, Allen Newell y Paul Rosenbloom. Este programa fue descrito en la revista SIGART de agosto de 1991 por John Laird, Mike Hucka y Scott Huffman del Artificial Intelligence Laboratory de la University of Michigan, y por Paul Rosenbloom del Information Sciences Institute de la University of Southern California. Se inició en 1983 y aún está en etapas de desarrollo, siendo la versión más reciente, la del año 2004, que está en uso en muchos países del mundo. El sistema incluye la creación de subobjetivos de aprendizaje que es equiparable al aumento de la cantidad de reglas de actuación y conceptos en la memoria del sistema calificado como inteligente; porque aprende en interacción con un entorno externo.

En el ámbito de la aritmética un programa informatizado específico basado en la arquitectura Soar es el denominado Mundo de la Sustracción - "The subtraction world", software que representa en una pizarra como hacer problemas de sustracción multicolumna. Incluye una versión revisada del procedimiento de VanLehn en Sierra, pero usando la arquitectura cognitiva Soar. El lenguaje fue desarrollado originalmente por Randolph M. Jones, Meter M. Wiemer-Hastings, y John E. Larid en el laboratorio de Inteligencia Artificial de la Universidad de Michigan. Posteriormente se crearon otras versiones por autores como Randolph M. Jones.

VanLehn en 1990, compara la arquitectura Soar con Sierra, e indica que los dos sistemas en parte sostenían el mismo objetivo que era la obtención de procesos erróneos; aunque las dos investigaciones se solaparon en tiempo, ambos sistemas poseen diferencias notables que a juicio de VanLehn hacen inclinar la balanza hacia Soar como una arquitectura más completa. La diferencia mayor entre la Sierra y Soar es que Soar especifica modelos más detallados de la cognición que Sierra.

Por tanto, la diferencia mayor entre Soar y Sierra es que el primero construye una arquitectura cognoscitiva general, basada la teoría ACT (Adaptive Control of Thought: Control Adaptativo del Comportamiento) de Anderson $(1982,1983)$. En esta teoría los mecanismos de aprendizaje están muy relacionados con el resto de los procesos, especialmente con el sistema de presentación de la información que funciona como arquitectura de aplicación a cualquier proceso informatizado de aprendizaje.

\section{Sistema informatizado - "HS (BUSCADOR HEURÍSTICO)”}

Diseñado por (Ohlsson, Ernst \& Rees, 1992). Muestra cómo la simulación puede permitir al diseñador analizar la interacción de la instrucción propuesta con el conocimiento anterior que posee el individuo. Es un simulador que muestra la parte conceptual o semántica del conocimiento del proceso de la sustracción. Los autores tomaron a cuatro estudiantes simulados, dos de los cuáles entendían 
significado conceptual de la sustracción y los otros dos no.

Ohlsson et al., encontraron que para los aprendices era más fácil entender el proceso de la sustracción cuando el estudiante simulado comprendía la semántica del sistema en base diez.

Además su trabajo, muestra cómo las simulaciones pueden revelar interacciones de instrucción con el conocimiento anterior. Esto es considerado como una valiosa información a nivel pedagógico.

Por tanto, si los simuladores que hemos descrito con anterioridad se centraban más bien en procesos sintácticos de la sustracción, el Sistema $H S$, toma como referencia el dominio conceptual del procedimiento como entrada al sistema.

$H S$, es un prototipo informatizado basado en reglas generales que descubren cuando se ejecutan conductas incorrectas en el ámbito de la sustracción.

Como hemos indicado en la descripción del sistema Soar, la diferencia mayor entre Soar y los otros dos modelos es que construye una arquitectura cognoscitiva general. Por el contrario, "HS" y Sierra buscaron desarrollar específicamente los aspectos del modelo de la sustracción y por tanto, sus sistemas son el resultado de implementar el desarrollo teórico empíricamente comprobado de una teoría que sustenta la adquisición del error aritmético.

Finalmente, estamos informados de la existencia de otros modelos como el de Ohlson y Langley denominado DPF (1985). Este sistema, busca el diagnóstico de los errores mediante el análisis del espacio del problema a resolver. En mayor o menor mediada, se basan en los que hemos descrito anteriormente.

\section{Conclusiones}

En resumen, la sustracción como modelo cognitivo procesal, se ha convertido en plataforma única, que ha contribuido al diseño y generación de sistemas inteligentes, aplicados a distintos ámbitos de la adquisición del conocimiento matemático. A lo largo del artículo, hemos analizado los prototipos de investigación informatizados más importantes en el diagnóstico de los errores que se producen durante el aprendizaje de la sustracción.

La finalidad última de estos sistemas, es el diagnóstico y catalogación de errores en el ámbito del conocimiento procesal. Podemos decir, que no sólo representan el proceso matemático sino que rastrean las acciones llevabas a cabo durante la tarea, para que el conjunto de pasos seguidos pueda volverse objeto de estudio y puedan especificarse habilidades necesarias para poder resolver el proceso. Esta característica, en definitiva, constituiría una de las aportaciones más sobresalientes a nivel pedagógico, puesto que el entorno del ordenador, propiamente estructurado, puede proporcionar una herramienta, poderosa, motivadora, y aún sin explotar que enfoque la atención del estudiante directamente en sus propios procesos del pensamiento matemático, (Collins and Brown, 1988). Del mismo modo, estos sistemas ayudan a centrar la atención del docente, en esos procesos de aprendizaje netamente abstractos que configuran el conocimiento matemático, ayudándole a comprender los pasos seguidos por el alumno en la formulación del error algorítmico.

\section{Referencias}

Anderson, J.R. (1982) Acquisition of cognitive skills. Psychological Review, 89, 369-406.

Anderson, J.R. (1983) The architecture of cognition, Harvard University Press: Cambridge, Massachusetts -EE.UU.

Brown, J. S., Burton, R.B. (1978) Diagnostic models for procedural bugs in basic mathematical skills. Cognitive Science, 2, 155-192. 
Brown,J.S. \& VanLehn, K. (1980) Repair Theory: A generative theory of bugs in procedural skills. Cognitive Science,4, 379-426.

Brusilovskiy, P. (1994) The Construction and Application of Student Models in Intelligent Tutoring Systems. Journal of Computer and Systems Sciences International, 32 (1), 70- 89.

Carbonell, J. R. (1970). AI in CAI: An artificial intelligence approach to computer assisted instruction. IA Systems, 11, 190-202.

Collins and Brown (1988). The computer as a Tool for Learning Through Reflection. In H. Mandl \& A. Lesgold (EDs.), Learning sigues for intelligent tutoring system (pp. 1-18).New York: SpringerVerlag.

Conati, C., VanLehn, K. (1996). POLA: A Student Modeling Framework for Probabilistic On-line Assessment of Problem solving Performance. In Proceedings of the Fifth Internacional Conference on User Modeling. Kailua-Kona, HI.

Dillenbourg P., Self J. (1992).A framework for learner modeling. Technical report AAI/AI-ED 74, Departament of Computing, Lancaster University.

Fritz, W. (1997). The world of substraction. Extraído en enero de 2004., de http://www.intelligentsystems.com.ar/intyst/fritzw.htm.

Newell, A., \& Simon, H. A. (1972). Human problem-solving. Englewood Cliffs, NJ: Prentice- Hall.

Ohlsson, S., \& Langley, P. (1985). Identifying solution paths in cognitive diagnosis (Tech. Rep. CMURITR -85-2). Pittsburgh, PA: Carnegie Mellon University, The Robotics Institute.

Ohlsson, S., \& Langley P. (1988). Psychological evaluation of path hypotheses in cognitive diagnosis. In H. Mandl \& A. Lesgold (EDs.), Learning sigues for intelligent tutoring system (pp. 42-62).New York: Springer-Verlag.

Olhlson, S, \& Rees, E. (1991). The function of conceptual understanding in the learning of arithmetic procedures. Cognition and Instruction, 103-180.

Ohlsson, S., Ernst, A.M. \& Rees, E. (1992). The cognitive complexity of doing and learning arithmetic. Journal for Research in Mathematics Education, 23, 5, 441-467

VanLehn, K. (1983a). Felicity conditions for human skill acquisition: Validating an AI-based Theory (Tech. Rep. No. CIS-21). Palo Alto, CA: Xerox PARC.

VanLehn, K. (1983b). On The representation of procedures in repair theory. In H.P. Ginsburg (Ed.), The development of mathematical thinking (pp.201-252). New York: Academic.

VanLehn, K. (1987). Learning one subprocedure per lesson. Artificial Intelligence, 31, 1-40.

VanLehn, K. (1990). Mind bugs: origins of procedural misconceptions. Cambridge, Mass.: MIT Press

Martin, J. D. and VanLehn, K. (1993). OLAE: Progress toward a multi--activity, Bayesian student modeller. In Brna, P., Ohlsson, S., and Pain, H., editors, Proc. of AI in Education (AI-ED 93), pages 410--417, Edinburgh, Scotland.

VanLehn, K., Ohlsson, S., \& Nason, R. (1994). Applications of Simulated Students: An Exploration. Journal of Artificial Intelligence in Education, 2, 135-175.

Young, R.M., \& O’Shea, T. (1981). Errors in children’s subtraction. Cognitive Science, 5, 153-177. 
(C) Ediciones Universidad de Salamanca 